\title{
ATUAÇÃO DO ENFERMEIRO NO REFERENCIAMENTO E CONTRARREFERENCIAMENTO DE PESSOAS COM AMPUTAÇÃO*
}

\author{
Micheli Leal Ferreira ${ }^{1}$, Mara Ambrosina de Oliveira Vargas ${ }^{2}$, Ana Maria Fernandes Borges Marques ${ }^{1}$, Andréa \\ $\mathrm{Huhn}^{3}$, Selma Regina de Andrade ${ }^{2}$, Caroline Porcelis Vargas ${ }^{4}$
}

\begin{abstract}
RESUMO: O objetivo foi identificar referenciamentos e contrarreferenciamentos realizados pelos enfermeiros da Rede de Atenção à Saúde, descrevendo sua atuação na assistência à pessoa com amputação. Estudo qualitativo, com coleta de dados realizada por entrevista semiestruturada com 21 enfermeiros dos três níveis de atenção à saúde de Florianópolis. A coleta ocorreu nos meses de abril e maio de 2015. Os dados foram organizados com auxílio do software ATLAS.ti 7.5.6. e analisados com base na técnica da Análise Temática. Emergiram duas categorias: Portas de entrada da pessoa com amputação; O enfermeiro na assistência e as implicações de sua atuação no referenciamento e contrarreferenciamento. Identificou-se como portas de entrada mais procuradas Unidades Básicas de Saúde, serviço de Emergência e o Centro Catarinense de Reabilitação. O foco inicial dos enfermeiros é oferecer melhor assistência à pessoa com amputação realizando referência e contrarreferência quando necessário.
\end{abstract}

DESCRITORES: Amputação; Enfermagem; Integralidade em saúde; Assistência à saúde.

\section{NURSING ACTIONS IN REFERENCE AND COUNTER-REFERENCE IN HEALTH CARE FOR PERSONS WITH AMPUTATION}

\begin{abstract}
The present study aimed to identify reference and counter-reference activities performed by nurses of the Health Care Network, by describing their actions in the delivery of care to persons with amputation. Qualitative study, with data collection performed by semi-structured interviews with 21 nurses of primary, secondary and tertiary levels of health care in Florianópolis. Data was collected in April and May 2015 and was organized with the aid of ATLAS.ti 7.5.6 software and analyzed with the use of Thematic Analysis technique. Two categories emerged: Entry points to the healthcare network (RAS) for persons with amputation, and Nurses role in care and the implications of the actions of these professionals in the reference and counter-reference system. Basic Health Units, Emergency services and the Rehabilitation Center of Santa Catarina (CCR) were identified as the most frequently accessed entry points. The primary focus of nursing professionals is to deliver higher quality care to persons with amputation through reference and counter-reference in health care when necessary.
\end{abstract}

DESCRIPTORS: Amputation; Nursing; Integral care in health; Health care.

\section{ACTUACIÓN DEL ENFERMERO EN EL SISTEMA DE REFERENCIA Y CONTRARREFERENCIA DE PERSONAS CON AMPUTACIÓN}

RESUMEN: El objetivo del estudio fue identificar los sistemas de referencia y contrarreferencia que describen la actuación de enfermeros de la Red de Atención a la Salud en la asistencia a la persona con amputación. Es un estudio cualitativo, cuyos datos fueron obtenidos por medio de entrevista semi estructurada con 21 enfermeros de los tres niveles de atención a la salud de Florianópolis en los meses de abril y maio de 2015. Las informaciones fueron organizadas con por medio del software ATLAS.ti 7.5.6. y analizadas con base en la técnica del Análisis Temático. Resultaron de eso dos categorías: Puertas de entrada de la persona con amputación; El enfermero en la asistencia y las implicaciones de su actuación en sistema de referencia y contrarreferencia. Se identificaron como puertas de entrada más buscadas Unidades Básicas de Salud, servicio de Emergencia y el Centro Catarinense de Rehabilitación. El infoque inicial de los enfermeros es ofrecer mejor asistencia a la persona con amputación, realizando referencia y contrarreferencia cuando sea necesario.

DESCRIPTORES: Amputación; Enfermería; Integralidad en salud; Asistencia a la salud.

*Artigo extraído da dissertação intitulada: “Referência e contrarreferência na atenção à saúde das pessoas com amputação na visão do enfermeiro: uma perspectiva bioética". Universidade Federal de Santa Catarina, 2015.

${ }^{1}$ Enfermeira. Doutoranda em Enfermagem. Universidade Federal de Santa Catarina. Florianópolis, SC, Brasil.

${ }^{2}$ Enfermeira. Doutora em Enfermagem. Docente de Graduação e Pós-Graduação em Enfermagem da Universidade Federal de Santa Catarina. Florianópolis, SC, Brasil.

${ }^{3}$ Tecnóloga em Radiologia. Doutoranda em Enfermagem. Docente do Instituto Federal de Santa Catarina. Florianópolis, SC, Brasil.

${ }^{4}$ Enfermeira. Mestranda em Enfermagem. Universidade Federal de Santa Catarina. Florianópolis, SC, Brasil.

Autor Correspondente:

Caroline Porcelis Vargas

Universidade Federal de Santa Catarina

R. Ruth Pereira 806 - 88058-640 - Florianópolis, SC, Brasil

E-mail:k2vargas@gmail.com
Recebido: 09/02/2017

Finalizado: $14 / 08 / 2017$ 


\section{INTRODUÇÃO}

A amputação é um recurso terapêutico da medicina tão antigo quanto a própria humanidade. Existem registros com mais de 1000 anos de amputações por deformidades congênitas; traumáticas como resultado de batalhas ou acidentes; e como tratamento para algumas doenças ou complicações. Atualmente, as amputações eletivas ocorrem para evitar complicações e, por conseguinte oferecer melhores condições de vida à pessoa, especialmente quando existe dificuldade de intervenção endovascular que impossibilite a preservação do membro afetado ${ }^{(1-2)}$.

Estudos que abordam o momento da amputação mencionam esta como um tratamento a partir de um trauma ocorrido, complicações da Diabetes Mellitus (DM), Hipertensão Arterial Sistêmica (HAS) e nos casos de infecção ${ }^{(3-5)}$.

Considerando a mudança do perfil epidemiológico que o Brasil vem apresentando desde 1950, as doenças crônicas não transmissíveis (DCNT) assumiram e continuam com papel de destaque entre as principais causas de morbimortalidade, trazendo a necessidade de apontá-las como problema de magnitude global e ameaça à saúde e ao desenvolvimento humano por gerarem diversas dependências e deficiências, muitas vezes decorrentes da amputação por complicações ${ }^{(6-7)}$.

O Censo Brasileiro de 2010 realizado pelo Instituto Brasileiro de Geografia e Estatística (IBGE) sinaliza que o montante de $24 \%$ de toda a população do país possui alguma deficiência, dentre estes, aproximadamente 3 milhões de brasileiros vivem com alguma deficiência de natureza motora ${ }^{(8)}$.

Diante da magnitude desta problemática, faz-se necessário o empoderamento e a consequente implementação integral das políticas públicas existentes. Neste sentido, destaca-se a integralidade da assistência "entendida como um conjunto articulado e contínuo das ações e serviços preventivos e curativos, individuais e coletivos, exigidos para cada caso em todos os níveis de complexidade do sistema" (9:40).

A Lei 8080/90 dispõe sobre o fluxo de atendimento do usuário, definindo que:

...as ações e serviços de saúde, executadas pelo Sistema Único de Saúde (SUS), seja diretamente ou mediante participação complementar da iniciativa privada, serão organizadas de forma regionalizada e hierarquizada em níveis de complexidade crescente ${ }^{(10: 5)}$.

A hierarquização do SUS ocorre para que o usuário tenha à sua disposição acesso universal, igualitário e ordenado, com ações e serviços de saúde que se iniciem nas portas de entradas do SUS, e exista continuidade na rede regionalizada e hierarquizada, de acordo com a complexidade do serviçO ${ }^{(11)}$.

A organização das Redes de Atenção à Saúde (RAS) se dá de maneira que o usuário tenha perto de sua casa um serviço da atenção primária à saúde para intervir nas situações mais casuais. Quando essas necessidades não podem ser resolvidas por completo neste setor, o usuário então é referenciado para um nível de maior complexidade para atendimento com especialistas ${ }^{(11)}$.

Como definição do processo de referência e contrarreferência, tem-se que referência representa o processo de encaminhar o usuário para um nível de maior complexidade e densidade tecnológica da RAS, enquanto a contrarreferência diz respeito ao mesmo processo, mas para retorno do usuário para um nível de menor grau de complexidade da $\operatorname{RAS}^{(12)}$.

Considerou-se a problemática da pessoa com amputação eletiva ou traumática, por reconhecer que esta precisa de cuidados de reabilitação. Enfatiza-se que, para isso, precisa dos diferentes níveis de atenção à saúde e que estes trabalhem em rede, evitando assim reinternações seguidas e a cronificação de processos agudos.

Optou-se por investigar esta temática na perspectiva dos enfermeiros, por terem importante papel de gestor do cuidado, além disso, têm compromissos éticos da profissão, e são responsáveis pelo cuidado específico e o acompanhamento de diversas doenças crônicas que são as principais causas nas amputações eletivas.

Logo, estima-se de extrema relevância contextualizar a atuação do enfermeiro no processo de assistência à saúde da pessoa com amputação em todos os níveis de atenção, a fim de tornar pública 
as dificuldades enfrentadas e a necessidade de investimentos na estruturação deste processo, uma vez que poderá direcionar ou amparar as ações destes profissionais.

Sendo assim, constituiu-se como questão de pesquisa desta investigação: como se dá a atuação do enfermeiro, na assistência à saúde da pessoa com amputação, nos três níveis da RAS do município de Florianópolis - estado de Santa Catarina (SC)?

Os objetivos do estudo foram: identificar os referenciamentos e contrarreferenciamentos realizados pelos enfermeiros em cada ponto da RAS; e descrever a atuação dos enfermeiros na assistência à saúde da pessoa com amputação.

\section{- MÉTODO}

Esta é uma pesquisa qualitativa, exploratória e analítica. Foi desenvolvida nos três distintos níveis de atenção à saúde: primária, secundária e terciária.

Os locais de coleta foram a Unidade de Internação Cirúrgica II (UIC II) do Hospital Universitário Professor Polydoro Ernani de São Thiago, hospital escola da Universidade Federal de Santa Catarina (UFSC/HU); o Centro Catarinense de Reabilitação (CCR); a Policlínica Municipal Continente; o Ambulatório do HU; e cinco Unidades Básicas de Saúde (UBS): UBS Centro; UBS Coqueiros; UBS Córrego Grande; UBS Ingleses; e UBS Saco dos Limões.

Usou-se como critério de escolha das UBS do município de Florianópolis aquelas com maior população de idosos (65 anos ou mais) atendida em cada distrito sanitário, devido à estreita relação entre as amputação eletivas com as doenças crônicas, comprovadamente mais frequentes neste grupo populacional.

Os participantes do estudo foram 21 enfermeiros, alocados de forma fixa nos locais do estudo. Foram excluídos os enfermeiros que possuíam menos de três meses de atuação na RAS de Florianópolis; que não prestaram assistência direta ou indireta à pessoa com amputação; e/ou que estavam afastados por férias ou licença de saúde no período da coleta de dados.

A seleção dos sujeitos foi aleatória e a coleta de dados ocorreu nos meses de abril e maio de 2015, por meio de entrevistas individuais semiestruturadas gravadas. Para as entrevistas foram utilizados três roteiros, um para cada nível de atenção devido a particularidades na assistência. Os roteiros foram validados por especialistas no assunto e, após transcritas, solicitou-se validação de conteúdo a cada um dos entrevistados.

Para auxiliar no tratamento e análise dos dados, as entrevistas foram importadas para o software ATLAS.ti 7.5.6 (Qualitative Research and Solutions). Todas as transcrições foram inseridas no formato Microsoft Word como documentos e constituíram os primary documents. A análise dos dados ocorreu com base na técnica da Análise Temática, que prevê como critérios a pré-análise, categorização, inferência, descrição e interpretação ${ }^{(13)}$.

Projeto aprovado pelo Comitê de Ética em Pesquisa via Plataforma Brasil, sob parecer $\mathrm{n}^{\circ} 970.902$ de 24/02/2015. O anonimato dos participantes foi assegurado mediante uso do codinome $\mathrm{E}$ (letra) seguido do número 1 ao 21 (E1, E2, E3... E21). A distribuição dos números se deu de forma aleatória.

\section{RESULTADOS}

Com foco em atender os objetivos deste estudo, emergiram dos resultados duas categorias temáticas: Portas de entrada da pessoa com amputação; O enfermeiro na assistência e as implicações de sua atuação no referenciamento e contrarreferenciamento.

\section{Portas de entrada da pessoa com amputação}


Os enfermeiros, participantes do estudo, sinalizaram as distintas portas de entrada da pessoa com amputação na RAS do município de Florianópolis. Conforme descrição a seguir, constata-se que a porta de entrada se difere conforme o momento e necessidade da pessoa com amputação.

Neste sentido, a porta de entrada mais procurada logo após amputação e alta hospitalar é a UBS, em sua grande maioria devido à necessidade de troca de curativo; seguida pelo Ambulatório do HU, entrada esta justificada pelo direcionamento da própria instituição durante a internação.

Eles chegam ao centro de saúde, depois da amputação, após internação no $H U$, não tiveram alternativa e tiveram o membro amputado. Neste primeiro momento eles procuram o posto por causa do curativo do coto. (E12)

Eles chegam via recepção. Mas, algumas demandas acabam vindo pelos agentes comunitários de saúde, ou solicitação de visita domiciliar por telefone! (E3)

No ambulatório do HU, elas chegam normalmente através das consultas das unidades de internação hospitalar, da emergência, da endocrinologia, ou às vezes da unidade básica de saúde. (E14)

Outra porta de entrada é o serviço de emergência do referido hospital, nos casos de amputações recorrentes ou complicações pós-operatórias.

As pessoas chegam de um posto de saúde, pelo ambulatório, ou por agudização ou dor, o médico faz uma carta de encaminhamento, eles entram pela nossa emergência e acabam passando por amputação. Ou o paciente vem direto para a emergência por causa da dor, nunca foi no posto, tanto que hoje me falaram: - A emergência parece uma unidade de vascular! (E18)

O Centro Catarinense de Reabilitação (CCR) foi identificado como porta de entrada nos casos em que o foco é a reabilitação e o processo protético. Entretanto, a demora na chegada deste usuário é preocupante, principalmente porque em inúmeras vezes estas pessoas não recebem atendimento nos outros pontos da RAS após a amputação.

Gostaria que elas chegassem mais cedo. [...] Os pacientes chegam aqui muito tempo depois, quase que sem orientação, sem preparação do coto. Eles poderiam levar menos tempo no preparo do coto, na protetização, se recebessem informação. (E16)

Uma vez que a pessoa com amputação ingressa na RAS do município, o enfermeiro deve identificar as necessidades específicas da cada usuário e prestar uma assistência individualizada. Descreve-se a seguir como se dá a atuação do enfermeiro na assistência à pessoa com amputação, assim que esta entra na RAS do município através das portas de entrada anteriormente apresentadas.

\section{Oenfermeironaassistênciaeasimplicações desuaatuaçãonoreferenciamentoecontrarreferenciamento}

Evidenciou-se que nas UBSs o enfermeiro tem como plano de ação um conjunto de atividades que incluem: identificar a causa da amputação; avaliar a situação do coto ou da ferida operatória se houver; quando necessário tratar doenças de base; apontar necessidades específicas a serem abordadas, sejam elas físicas ou psicossociais, considerando a reabilitação, a prevenção de agravos e a inclusão social desta pessoa; por fim, realizar e/ou auxiliar nos respectivos referenciamentos.

Primeiro teria que avaliar a amputação, o motivo desta amputação, se ele tinha conhecimento da sua doença, dos cuidados que tinha que ter com os pés dele, se é alguma coisa que foi contínua ou se agravando, se foi uma situação traumática. E depois a reabilitação. (E10)

Procuramos inserir eles nos grupos, e dentro da capacidade deles, estimulamos a manterem a qualidade de vida, mesmo porque amputou um membro e depois poderá amputar outro. (E7)

Quando a pessoa com amputação chega ao Ambulatório do HU, o enfermeiro tem como objetivos identificar quaisquer sinais de alteração neurológica ou circulatória; possíveis lesões; encaminhar para solicitação de sapatos especiais, órteses ou próteses; e desempenhar atividades educativas de prevenção. Quando este profissional se depara com lesões, ou situações desfavoráveis de cicatrização, sua orientação é que esta pessoa vá diretamente para o serviço de emergência: 
Primeiro avalio para ver se ele está com alteração neurológica ou circulatória, mesmo que tenha feito amputação, fazemos para evitar outras amputações. [...] Na reabilitação, dão calçados especiais, órtese dentro do sapato, ele caminha de uma forma diferente, vai mudando a estrutura, formando calosidades em outros locais do pé. Converso com o médico, preencho, e encaminho o paciente para o sapato especial e até se precisar de uma órtese é encaminhado por lá. [...] Às vezes eles me ligam e vejo se posso atender ou se tem que ir direto para a emergência para desbridar ou amputar, depois volta comigo para fazer o papel educativo e preventivo. (E14)

Com relação à atuação do enfermeiro na assistência à saúde da pessoa com amputação, em uma Unidade de Internação hospitalar do Nível Terciário de Saúde (NTS), os participantes da unidade verbalizaram que:

Se o paciente tem uma boa mobilidade, força física e condições de se locomover com um andador, orientamos e auxiliamos. Oferecemos uma cadeira de rodas para levar o paciente até o banho ou o vaso sanitário. [...] O paciente sai daqui com a consulta de retorno agendada e com uma prescrição de curativo. Acho muito pouco! (E20)

Na alta, orientamos os cuidados com o coto. Saem com uma pré-consulta com o ambulatório de curativos e retorno para uma consulta com a equipe da cirurgia vascular. Ensinamos como fazer o curativo em casa, mas se for um curativo que exige mais cuidado, orientamos que eles façam no posto de saúde, e se tiver qualquer intercorrência retornar na emergência do hospital. (E19)

Ainda no NTS, existe o CCR, onde o enfermeiro age nos tratamentos de lesão de pele, nos procedimentos ou controle de comorbidades, ampliando suas ações para atividades de educação em saúde visando à prevenção.

Quando eles chegam é para o tratamento de ferida e não mais para prevenção, embora trabalhamos a prevenção dali pra frente. [...] O cuidado da enfermagem, o nosso carro chefe, não é o curativo, mas a atividade de educação em saúde. (E16)

Quando a pessoa com amputação é acompanhada pela UBS e precisa de intervenção ou avaliação de um especialista, que não faça parte da equipe do Núcleo de Apoio de Saúde à Família (NASF), o encaminhamento é realizado pelo enfermeiro e/ou pelo médico.

Nos casos de protetização, o referenciamento é direcionado para o CCR, nas situações em que há necessidade de atendimento especializado (enfermagem, vascular, endocrinologia), o encaminhamento é para o atendimento no Nível Secundário de Saúde (NSS), no ambulatório do HU ou nas Policlínicas do município.

Não foram evidenciados relatos sobre encaminhamentos pós-amputação para acompanhamento da ferida operatória ou coto com os enfermeiros nas policlínicas, apesar da RAS de Florianópolis contar com um consultório referência em feridas e com atuação fixa da enfermagem. Sinaliza-se que, na prática, este não faz parte do fluxo de atendimento à pessoa com amputação, conforme apresentado a seguir:

Normalmente elas não chegam por uma amputação, mas encaminhadas para alguma consulta com especialistas como o vascular. Às vezes, recebemos paciente com amputação prévia na sala de feridas para fazer curativo. Não há referenciamento de paciente com amputação aqui nessa policlínica. (E15)

Quando a pessoa com amputação chega para uma consulta no Ambulatório do HU e está com uma ferida infectada ou com cicatrização desfavorável, é realizado o direcionamento para o NTS, através do próprio serviço de emergência.

Muitas coisas aqui no HU já estão institucionalizadas, mas tem outras que dependem realmente de conversarmos com o profissional. [...] Colocamos claramente para os centros de saúde que quando é lesão ou algum problema maior, deve ser encaminhado para emergência, porque se vier no ambulatório e eu identificar a lesão, encaminharei para a emergência. (E14)

Mas os profissionais de enfermagem da unidade de internação do HU afirmam ter pouco envolvimento com a contrarreferência da pessoa com amputação. Realizam em seletas vezes contatos com UBS, tendo com foco exclusivo o curativo e/ou a ferida operatória; no que diz respeito à reabilitação, 
ficam responsáveis outros profissionais conforme relatos:

Vejo muita dificuldade no processo de alta. A psicologia e o serviço social fazem um serviço legal [...] a assistente social orienta a parte da reabilitação, diz como ele faz para conseguir as próteses, fala do centro de reabilitação. Então dessa parte eu nada sei [...] Quando dá, faço contrarreferência para a atenção primária, passo orientações para o curativo. Como não temos um formulário pronto para isso, já fiz uma carta e liguei para informar sobre a situação do paciente. Mas isso não é padronizado! (E18)

Os enfermeiros que atuam no CCR verbalizam que realizam encaminhamentos para outro profissional do NTS nos ambientes hospitalares, quando há necessidade de alguma intervenção ou avaliação; contrarreferências para UBS quando se trata de moradores de outros municípios que continuarão o tratamento em casa; ou ainda, reencaminhamentos para o programa de protetização da referida instituição nos casos em que a pessoa com amputação está apta para retomar o processo.

Se o paciente chega com algum tipo de necrose ou suspeita de necessidade de outra amputação, encaminhamos para o hospital da cidade de origem ou para o hospital da regional. [...] Orientamos por escrito e verbalmente, fornecemos materiais para iniciar os cuidados com feridas e encaminhamos para continuidade dos mesmos na UBS do município. [...] Reencaminhamos para o programa de protetização do CCR, porque se ele tem o desequilíbrio, ele sai do programa para depois retornar. (E17)

Verificada a impossibilidade de resolução em um caso específico, os enfermeiros do CCR esquematizam ações e encaminhamentos necessários para que a pessoa com amputação possa se reestabelecer e futuramente retornar ao programa para concluir o processo de protetização.

\section{DISCUSSÃO}

Evidenciou-se que a pessoa com amputação utiliza como portas de entrada principalmente as UBSs, o serviço de emergência e o CCR, conforme a sua necessidade específica. Quanto à UBS, nota-se que a preocupação do usuário é o tratamento da ferida ou coto; o foco é a troca do curativo. Em nenhuma das falas percebeu-se articulação da UBS aos questionamentos quanto à reabilitação ou direitos da pessoa com amputação. Entretanto, sabe-se que a reabilitação deve ser trabalhada, sempre que possível, antes mesmo do procedimento da amputação, e que apesar deste ser um procedimento realizado pelos níveis de maior complexidade, a Atenção Primária de Saúde (APS) possui estreita relação com ele, além do importante papel de coordenadora do cuidado ${ }^{(14-15)}$.

O processo de trabalho do enfermeiro nas UBSs tem como premissas a relação interdisciplinar e multidisciplinar da equipe, assim como o incremento de ações preventivas, educativas e administrativas. Entretanto, ainda identificam-se atividades individuais e curativistas voltadas para o agravo de saúde do cliente, sendo assim submetido a uma realidade que exige ações individualizadas, tanto na assistência quanto na supervisão, no planejamento das ações e na gerência dos serviços ${ }^{(16)}$.

É a APS que elabora, acompanha e organiza o fluxo dos usuários entre os distintos pontos de atenção, além de oferecer cuidado através de uma relação horizontal, contínua e integrada através dos processos de referência e contrarreferência ${ }^{(16)}$. Esta afirmação vai ao encontro do que se revelou quanto à atuação do enfermeiro da UBS e suas implicações no processo de referência e contrarreferência; nas falas, notou-se a preocupação deste profissional em prestar um atendimento integral e contínuo.

A pessoa com amputação tende a procurar pela emergência hospitalar, como consequência de evento agudo com base nas doenças crônicas tão comuns nesta população, ou pelas complicações com a cicatrização do coto e/ou ferida. Este fato é aceitável uma vez que, conforme estabelecido nas diretrizes clínicas baseadas em evidências, os hospitais têm, como função, responder às condições agudas ou aos momentos de agudização das condições crônicas ${ }^{(12)}$.

Ainda, os relatos evidenciaram que nas unidades hospitalares encontram-se as pessoas que se submeteram às amputações recentes ou recorrentes. Os profissionais de enfermagem envolvidos com este fluxo relataram pouco envolvimento e conhecimento quanto ao processo de reabilitação e aos encaminhamentos. Estudos comprovam que intervenções precoces e intensivas por um enfermeiro qualificado para dar instruções, apoiar e auxiliar a pessoa com amputação, ajuda a diminuir significativamente a taxa de complicações e amputação entre os pacientes em alto risco, bem como 
otimiza os resultados nos processos de reabilitação ${ }^{(17-18)}$.

Muitos profissionais da alta complexidade desconhecem a referência para a reabilitação, repassando tal atividade de encaminhamento e orientação para os profissionais do serviço social e da fisioterapia, o que evidencia a fragmentação do cuidado ${ }^{(19)}$. Acredita-se que, para enfrentar esta situação, há de se criar um fluxo entre os serviços e pontos de atenção com referenciamentos e contrarreferenciamentos previamente estabelecidos. O objetivo é que as pessoas tenham acesso rápido e fácil, logo após a alta hospitalar, às UBSs mais próximas de seus locais de moradia, bem como contem com serviços de Reabilitação Física e Apoio Psicossocial(20).

A pessoa submetida à amputação vive um processo de transição que comporta distintas e severas mudanças, no qual necessita de adequação. De modo geral, a melhor adaptação está relacionada ao processo de assistência recebido pela pessoa, pois a existência de uma equipe atuando de forma interdisciplinar pode tornar a recuperação e o processo de reabilitação mais satisfatório ${ }^{(21-22)}$.

Então, se a pessoa com amputação não recebe o devido direcionamento, isso acarreta em sequelas bastante expressivas para si e sociedade, uma vez que a reabilitação tem como objetivo imediato o de proporcionar à pessoa com amputação um estilo de vida tão funcional quanto possível ${ }^{(23)}$.

O estudo teve como limitações o acesso aos profissionais dos diferentes níveis de atenção que já tivessem atuado com pessoas com amputação, uma vez que o registro de enfermeiros não nos informa sobre onde as pessoas com amputação foram atendidas ou por quais profissionais.

\section{CONSIDERAÇÕES FINAIS}

Ao identificar e descrever como se dá a atuação do enfermeiro em todos os pontos da RAS, bem como as implicações no processo de referenciamentos e contrarreferenciamentos das pessoas com amputação, como parte constituinte e integrante da assistência, exalta-se que a prevenção de doenças e agravos esteve presente em todos os níveis de atenção à saúde.

Além disso, ficou evidenciado que todas as premissas de uma RAS integrada e articulada giram em torno da conectividade dos distintos pontos, atuando em consonância para prestar atendimento integral, e que isso se dá pelo deslocamento efetivo entre os níveis de atenção à saúde.

O enfermeiro tem um importante papel de gestor do cuidado e deve atuar com o princípio da responsabilidade na promoção da saúde. No entanto, a integralidade e a continuidade na assistência à saúde da pessoa com amputação vêm sendo prejudicadas pela comunicação ineficaz entre os distintos níveis de atenção, e isso reflete em um processo de reabilitação demasiadamente demorado e caro para o SUS.

O percurso da pessoa com amputação nas RAS do município de Florianópolis mostrou que o CCR é o único ponto de referência para protetização no município e ela chega de forma tardia e sem as orientações necessárias.

Há necessidade de se padronizar as ações no que diz respeito à reabilitação e a assistência à saúde da pessoa com amputação, bem como criar instrumentos de amparo às ações não só do enfermeiro, mas de todos os profissionais envolvidos a fim de direcionar e facilitar as ações e o acesso a este usuário do SUS.

Considera-se necessário que outros estudos nesta linha de investigação sejam realizados, para amparar lacunas de capacitação, definir um fluxo de atendimento para a pessoa com amputação e fortalecer o processo de referência e contrarreferência na RAS.

\section{REFERÊNCIAS}

1. Jacobs C, Siozos P, Raible C, Wendl K, Frank C, Grützner PA, et al. Amputation of a lower extremity after severe trauma. Oper Orthop Traumatol. [Internet] 2011;23(4) [acesso em 14 jun 2015]. Disponível: http://dx.doi. org/10.1007/s00064-011-0043-9. 
2. Chalya PL, Mabula JB, Dass RM, Ngayomela IH, Chandika AB, Mbelenge N, et al. Major limb amputations: a tertiary hospital experience in northwestern Tanzania. J Orthop Surg Res. [Internet] 2012;(7) [acesso em 10 jun 2015]. Disponível: http://dx.doi.org/10.1186/1749-799X-7-18.

3. Slim H, Tiwari A, Ahmed A, Ritter JC, Zayed H, Rashid H.. Distal versus ultra distal bypass grafts: amputation-free survival and patency rates in patients with critical leg ischaemia. Eur J Vasc Endovasc Surg. [Internet] 2011;42(1) [acesso em 4 jun 2015]. Disponível: http://dx.doi.org/10.1016/j.ejvs.2011.03.016.

4. Dunkel N, Belaieff W, Assal M, Corni V, Karaca Ş, Lacraz A, et al. Wound dehiscence and stump infection after lower limb amputation: risk factors and association with antibiotic use. J Orthop Sci. [Internet] 2012;17(5) [acesso em 30 mai 2015]. Disponível: http://dx.doi.org/10.1007/s00776-012-0245-5.

5. Moxey PW, Hofman D, Hinchliffe RJ, Poloniecki J, Loftus IM, Thompson MM, et al. Delay influences outcome after lower limb major amputation. Eur J Vasc Endovasc Surg. [Internet] 2012;44(5) [acesso em 4 jun 2015]. Disponível: https://doi.org/10.1016/j.ejvs.2012.08.003.

6. Marinho MGS, Cesse EAP, Bezerra AFB, de Sousa IMC, Fontbonne AF, de Carvalho EF. Análise de custos da assistência à saúde aos portadores de diabetes melito e hipertensão arterial em uma unidade de saúde pública de referência em Recife - Brasil. Arq Bras Endocrinol Metab. [Internet] 2011;55(6) [acesso em 2015 jun 14]. Disponível: http://dx.doi.org/10.1590/S0004-27302011000600007.

7. de Souza CF, Gross JL, Gerchman F, Leitão CB. Pré-diabetes: diagnóstico, avaliação de complicações crônicas e tratamento. Arq Bras Endocrinol Metab. [Internet] 2012;56(5) [acesso em 10 jun 2015]. Disponível: http://dx.doi. org/10.1590/S0004-27302012000500001.

8. Instituto Brasileiro de Geografia e Estatística (IBGE). Censo Demográfico 2010: Características gerais da população, religião e pessoas com deficiência [Internet] 2013 [acesso em 14 jun 2015]. Disponível: http://www. ibge.gov.br/home/estatistica/populacao/censo2010/caracteristicas_religiao_deficiencia/caracteristicas_religiao_ deficiencia_tab_gregioes_xls.shtm.

9. Ministério da Saúde (BR). Secretaria de Atenção à Saúde. HumanizaSUS: Cartilhas da política nacional de humanização. Brasília: Ministério da Saúde; 2009.

10. Brasil. Lei n. 8080 de 19 de Setembro de 1990. Dispõe sobre as condições para a promoção, proteção e recuperação da saúde, a organização e o funcionamento dos serviços correspondentes e dá outras providências. Diário Oficial da República Federativa do Brasil, Brasília, 20 set. 1990.

11. Ministério da Saúde (BR). Decreto n. 7.508, de 28 de junho de 2011. Regulamenta a Lei n. 8.080, de 19 de setembro de 1990, para dispor sobre a organização do Sistema Único de Saúde - SUS, o planejamento da saúde, a assistência à saúde e a articulação interfederativa, e dá outras providências. Diário Oficial da União, Brasília, 28 jun. 2011.

12. Mendes EV. As redes de atenção à saúde. Brasília: Organização Pan-Americana da Saúde; 2011.

13. Minayo MCS. O desafio do conhecimento: pesquisa qualitativa em saúde. 16ª ed. São Paulo: Hucitec; 2014.

14. Mendes EV. O cuidado das condições crônicas na atenção primária à saúde: o imperativo da consolidação da estratégia da saúde da família. Brasília: Organização Pan-Americana da Saúde; 2012.

15. Ministério da Saúde (BR). Secretaria de Atenção à Saúde. Diretrizes de atenção à pessoa amputada. $1^{\mathrm{a}}$ ed. Brasília: Editora do Ministério da Saúde; 2013.

16. Freitas GM, Santos NSS. Atuação do enfermeiro na atenção básica de saúde: revisãointegrativa de literatura. R. Enferm. Cent. O. Min. [Internet] 2014;4(2) [acesso em 21 fev 2017]. Disponível: http://dx.doi.org/10.19175/ recom.v0i0.443.

17. Ren M, Yang C, Lin DZ, Xiao HS, Mai LF, Guo YC, et al. Effect of intensive nursing education on the prevention of diabetic foot ulceration among patients with high-risk diabetic foot: a follow-up analysis. Diabetes Technol Ther. [Internet] 2014;16(9) [acesso em 4 ago 2015]. Disponível: http://dx.doi.org/10.1089/dia.2014.0004.

18. Price B, Moffatt B, Crofts D. Managing patients following a lower limb amputation. J. Comp. Neurol. [Internet] 2015;29(3) [acesso em 10 ago 2016]. Disponível: https://www.jcn.co.uk/files/downloads/articles/jcn-06-2015-10managing-patients-following-a-lower-limb-amputation.pdf. 
19. Vargas MAO, Ferrazzo S, Schoeller SD, Drago LC, Ramos FRS. Rede de atenção à saúde à pessoa amputada. Acta paul. enferm. [Internet] 2014;27(6) [acesso em 10 jun 2015]. Disponível: http://dx.doi.org/10.1590/19820194201400086.

20. Ministério da Saúde (BR). Secretaria de Atenção à Saúde. Política Nacional de Saúde da Pessoa Portadora de Deficiência. Brasília: Editora do Ministério da Saúde; 2012.

21. Cruz DM, do Nascimento LRS, da Silva DMGV, Schoeller SD. Red de apoyo para las personas con discapacidad. Cienc. enferm. [Internet] 2015;21(1) [acesso em 28 abr 2016]. Disponível: http://dx.doi.org/10.4067/S071795532015000100003.

22. Fortington LV, Rommers GM, Wind-Kral A, Dijkstra PU, Geertzen JH. Rehabilitation in skilled nursing centres for elderly people with lower limb amputations: a mixed-methods, descriptive study. J Rehabil Med. [Internet] 2013;45(10) [acesso em 3 ago 2015]. Disponível: http://dx.doi.org/10.2340/16501977-1210.

23. Brito TDQ, de Oliveira AR, Eulálio MC. Disability and aging: study of social representations of the elderly from physical therapy rehabilitation. Av. Psicol. Latinoam. [Internet] 2015;33(1) [acesso em 25 abr 2016]. Disponível: http://dx.doi.org/10.12804/apl33.01.2015.09. 